\title{
Ein Purist auf Abwegen
}

\section{Pierre Passett (Zürich)}

Zusammenfassung: Das irreführende Freudsche Diktum vom reinen Gold der Psychoanalyse und den Legierungen der Psychotherapie hat die Psychoanalytiker immer wieder dazu bewogen, angestrengt die von ihnen idealisierte Psychoanalyse rein zu erhalten. Als Folge davon mussten diejenigen, die die Analyse in der Theorie und Praxis mit der Psychotherapie vermischt haben, sich den puristischen Vorwurf der Verwässerung bzw. Befleckung der reinen Lehre gefallen lassen. Dies ist widersinnig, denn Psychoanalyse und Therapie sind in weiten Bereichen annähernd identisch. Eben deshalb aber ist es notwendig, die tatsächlich vorhandenen, relevanten Unterschiede zu sehen, ernst zu nehmen und die richtigen Konsequenzen aus ihnen für Praxis und Lehre zu ziehen. Mit der Feststellung, es handle sich bei dieser Unterscheidung um eine falsche Alternative, drückt man sich lediglich um eine Stellungnahme. Es geht nicht darum, Unterscheidungskriterien zu definieren, wie das die IPA versucht. So etwas läuft nur darauf hinaus, durch willkürliche Herstellung von Differenzen zwei Dinge auseinanderzudividieren und künstlich die Lehre rein zu halten. Es kommt im Gegenteil darauf an, schlicht und einfach, die bestehenden Unterschiede zu sehen, also Differenzen anzuerkennen, nicht solche herzustellen. Die Anerkennung solcher Differenzen erlaubt uns, das, was wir im jeweiligen Falle tun, genauer zu situieren und gibt uns selbst und unseren Patienten die Möglichkeit zu entscheiden, in welcher Richtung die Prozesse, die wir miteinander unternehmen, gehen sollen: mehr Richtung Psychotherapie oder Richtung Psychoanalyse.

Schlüsselwörter: Aktualität der Psychoanalyse; Autonomie von Analysand und Analytiker; Bewusste und unbewusste Übertragung; Einsicht und Veränderung; Suggestion; Therapeutische Wirkung und Selbsterfahrung; Unabgegrenztheit der menschlichen Psyche; Ursachen und Motive im psychischen Leben.

Im Vortrag, der diesem Aufsatz zu Grunde liegt, habe ich als Ausgangspunkt meiner Überlegungen die Darstellung zweier Fälle benutzt, die je einem der beiden Idealtypen (Psychotherapie/Psychoanalyse) sehr nahe kommen. Aus Gründen 
der Diskretion verzichte ich in der hier publizierten Version auf die detaillierte Darstellung dieser zwei Fallgeschichten und begnüge mich damit, kursorisch mitzuteilen, worum es in den beiden ging. Es ist mir bewusst, dass dies eine grosse Einbusse an Anschaulichkeit mit sich bringt, da es sich aber um die nachträgliche Dokumentation eines Vortragszyklus handelt, halte ich eine solche Veränderung für vertretbar.

Im ersten Fall ging es um eine sich über 18 Stunden erstreckende Kurztherapie einer fünfundzwanzigjährigen Frau, die an plötzlich aufgetretenen, medizinisch nicht begründbaren Schwindelanfällen und Angst vor diesen Anfällen litt, sowie an gelegentlich auftretenden Tachikardien. Da im Erstgespräch neben dieser Symptomatik eher zwischen den Zeilen eine Reihe von grösseren und kleineren Lebenslügen sichtbar und erahnbar wurden, mit denen sich diese junge Frau über ihre tatsächliche Lebenssituation hinwegschummelte, schlug ich ihr vor, unter Berücksichtigung der Doppelbedeutung des Wortes Schwindel diese Anfälle so zu verstehen, dass sie in symptomatischer Weise einerseits einen Hinweis auf alle diese nicht zur Kenntnis genommenen Tatsachen in ihrem Leben bedeuteten, diese also zur Kenntnis bringen wollten, andererseits diese Fakten aber gerade wieder mit dem Schleier des Nichtwissens, wofür ja die Ohnmacht in den Anfällen steht, zudeckten. Es handelte sich bei diesen verleugneten «facts of life», wie sich im Verlaufe der Behandlung zeigte, um Illusionen über ihre angebliche Zufriedenheit mit ihrer Partnerbeziehung und ihrer Arbeit, massive, aber nicht zugegebene Vorwürfe gegen ihren Partner und dessen parasitäres Verhalten ihr gegenüber, eine nur scheinbar völlig überwundene Kokainsucht, ignorierte Unstimmigkeiten im Verhältnis zu ihrer Herkunftsfamilie, verleugnete Ressentiments und Aggressionen gegen Arbeitskolleginnen, uneingestandene sexuelle Wünsche und geflissentlich übersehene Differenzen zwischen ihrem tatsächlichen Lebensstil und ihren diesbezüglichen Wünschen. Ich stellte ihr in Aussicht, dass in den maximal 20 Stunden der Therapie diese weggeschwindelten Dinge, auf die sie in der Vorbesprechung nicht explizit eingegangen war, von selbst zur Sprache kommen würden, was möglicherweise zur Folge haben werde, dass dann die Anfälle und die Angst vor ihnen ausbleiben würden. Diese suggestive Prognose führte dazu, dass die Patientin, immer scheinbar zufällig oder contre cœur, in den 18 nachfolgenden Stunden auf diese Dinge zu sprechen kam, und sich, unterstützt durch meine Fragen, Anregungen und Interpretationen mit ihnen auseinanderzusetzen begann. Die Schwindelanfälle und die Angst vor ihnen verschwanden schon nach der dritten Sitzung und die etwas später erst zur Sprache gebrachten Tachikardien ebenfalls umgehend, nachdem ihr Zusammenhang mit unterdrückten «bösen» Gedanken 
gegenüber von ihr insgeheim gering geschätzten Arbeitskolleginnen aufgedeckt worden war.

Beim zweiten Fall handelt es sich um die Behandlung eines 40-jährigen Prokuristen, der über mehr als sieben Jahre wöchentlich einmal zu mir kam und auf der Couch liegend frei assoziierte. Die niedere Frequenz war vor allem dadurch bedingt, dass er in einer anderen Stadt wohnte und eine lange Anreise hatte. Anlass zur Analyse war, dass er mit 40 Jahren noch nie einen sexuellen Kontakt zu einem anderen Menschen gehabt hatte und dass er, wie er selber betonte, unabhängig davon, ob sich das würde ändern können, wissen wollte, warum das so gekommen war. Die ganze Analyse drehte sich dann in einer weiten Spirale um eine bizarr erscheinende, zentrale Onaniephantasie. In dieser spielte ein Gebrechen der vorgestellten weiblichen Sexualpartnerin eine Rolle, dessen Funktion es war, einerseits die Strafe für die sexuelle Verführung durch die imaginierte Frau darzustellen und andererseits den Rachedurst der extrem überprotektiven, ihn bis ins Erwachsenenalter vor fast allen sozialen Kontakten abschirmenden Mutter zu stillen. So war dieses Gebrechen in einer höchst komplexen Weise jenes Attribut der sexuellen Szene, welches die Erfüllung von Triebwünschen und die Abwehr derselben in einem verwirklichte. Nachdem eine eher zufällige Frage von mir diese Onaniephantasie zum ersten Mal ins Gespräch gebracht hatte, verpasste der Analysand mehrmals auf dem Weg zu mir die richtige Bussstation zum Aussteigen. Die langwierige, immer wieder ins Stocken geratende Analyse dieser hier extrem vereinfacht wiedergegebenen Zusammenhänge bildete eine Art Hintergrund, vor welchem er langsam tastend reale sexuelle Beziehungen aufzunehmen begann, zuerst in einem später sich als Irrtum herausstellenden homosexuellen coming out, dann mit einer Reihe psychisch unstabiler Frauen, von denen er mit keiner während der Zeit der Behandlung je eine befriedigende und andauernde Beziehung zustande brachte. Trotzdem erlebte dieser Mann die Analyse als eine beglückende Erfahrung, weil sie ihm Einblicke in das dunkle Labyrinth seiner Seele ermöglicht hatte. Während das Geständnis der peinlichen Onaniephantasie in diesem Falle als Antwort auf eine von mir absichtslos und ohne Not gestellte Frage erfolgte (ob er selbst einmal ein Gebrechen wie dasjenige des Vaters, von dem er zuvor erzählt hatte, gehabt habe), waren die Enthüllungen der ersten Patientin Reaktionen auf die mit strategischer Absicht von mir gestellte Frage nach ihren Lebenslügen.

Was ist der für unser Thema relevante Unterschied zwischen dem ersten Fall, den ich als Therapie, genauer eine Variante von Fokaltherapie bezeichnen würde und dem zweiten, den ich für eine Analyse halte. Wenn ich mir die Frage 
stelle, was wohl den ersten Schwindelanfall der oben beschriebenen Patientin ausgelöst haben mag, so muss ich zugeben, dass ich keine Ahnung habe und mich mit der Vermutung zufrieden gebe, es sei wohl ein banales somatisches Moment, wie etwa ein plötzlicher Blutdruckabfall gewesen. Sicher hatte sie den Anfall nicht, weil ihr Leben gespickt mit grösseren und kleineren Lebenslügen war. Kann man aber wenigstens sagen, dass die Lebenslügen und die uneingestandenen sexuellen Wünsche schuld daran waren, dass die Angst vor dem Schwindel, der sich aus unbekannten Gründen eingestellt hatte, sich danach festsetzte? Auch darauf würde ich meinen Kopf nicht verwetten, denn ich kann grundsätzlich nichts Ungewöhnliches dabei finden, wenn jemand, der einmal in Ohnmacht gefallen ist und sich dabei verletzt hat, Angst hat, das könnte wieder passieren. Die Entstehung eines solchen Symptoms hat etwas relativ Zufälliges und Unauffälliges, aber das ändert nichts daran, dass es, wenn es einmal da ist, eine harte Realität ist, eine arge Störung, die man wieder los werden möchte. Die Frage, wie werde ich mein Symptom los, oder sonst eine Verhaltens- oder Erlebnisweise, die nicht zu mir passt oder mir nicht passt, das ist die Frage, die einen Menschen in eine Therapie führt. Und diese Frage hat der Therapeut ernst zu nehmen und er sollte idealerweise über ein Repertoire von Handlungen, Interventionen, Vorkehrungen usw. verfügen, die mit einer gewissen Wahrscheinlichkeit dazu führen können, dass das Symptom des Patienten verschwindet, dass sich bei diesem eine Veränderung einstellt in dem Sinne, den er wünscht. Im Allgemeinen geht das so vor sich, dass der Therapeut den Patienten zuerst davon überzeugen muss, dass sein Symptom ihn nicht einfach affiziert hat, wie eine somatische Krankheit, sondern dass es in irgend einer Weise auch Resultat eigenen Denkens und Handelns des Patienten ist und dass dieser in Folge dessen nur durch Eigenaktivität diesen Zustand verändern kann. Hat er den Patienten erst einmal davon überzeugt, leistet er entsprechend seiner therapeutischen Ausrichtung mehr oder weniger Hilfestellung beim Realisieren der Veränderung.

Ich will also eine Veränderung bei mir herbeiführen, in meinem Erleben, meinem Handeln, fühle mich aber selbst dazu nicht imstande. Also gehe ich zu jemandem, der mir dabei helfen kann. Ein solcher Gang hat nur einen Sinn, wenn ich davon ausgehe, dieser andere könne mich beeinflussen. Und zwar soll er mich besser beeinflussen können als jene, die es schon versucht haben. Denn mein Freund, meine Mutter, mein Arzt, sie haben mir ja alle schon gesagt, Du brauchst keine Angst zu haben, Dir fehlt nichts, das passiert Dir nicht wieder, das ist Nichts. Aber es hat nichts genützt. Das, wodurch eine Psychotherapie wirkt, ist psychische Beeinflussung und dafür haben wir einen Begriff, nämlich Suggestion. 
Diesen Begriff, Sie wissen es, meiden die Psychoanalytiker wie der Teufel das Weihwasser und auch die Psychotherapeuten anderer Couleur fühlen sich unbehaglich, wenn die Rede auf ihn kommt. Suggestion hat etwas Genantes, sie liegt nahe bei Manipulation, riecht politisch unkorrekt nach unerlaubtem Eingriff in die Autonomie des anderen. Der Psychotherapeut findet sich notwendig und nicht zufällig stets in einem unlösbaren Paradox gefangen, nämlich, bei gleichzeitiger Wahrung seiner und des Patienten Autonomie, doch so in des Patienten Leben einzugreifen, dass sich darin etwas verändert. Freud hatte in dem ihm eigenen aufklärerischen Enthusiasmus zunächst angenommen, Einsicht in dieWahrheit der Lebensgeschichte habe automatisch einen verändernden Effekt. Die Behandlung der Hysterie nährte eine Weile lang diesen Glauben, weil es sich oft genug ergab, dass die Einsicht in die im hysterischen Symptom inszenierten unbewussten Gedanken, die Inszenierung hinfällig werden liess.

Leider gilt dies nur für die Hysterie, welche man auch als eine unbewusste Simulation bezeichnen könnte, aber nicht einmal dort immer und schon gar nicht für alles übrige psychische Leiden. Nachdem auch die Entdeckung der Übertragung und des Widerstandes, so wie die technische Forderung des Durcharbeitens den therapeutischen Erfolg nicht garantieren konnten, verfiel Freud immer mehr in jenen wohlbekannten therapeutischen Pessimismus, den seine Anhänger meist ignorierten, oder mittels Deutungen entschärften. Ob dieser «Pessimismus» nicht vielmehr einfach Ausdruck seines Unwillens war, überhaupt Therapie zu betreiben, ist eine Frage, der nachzugehen, sich lohnen würde. Jedenfalls haben sich Freuds Anhänger seltsamerweise niemals gross daran gestört, dass seine wenigen publizierten Fallgeschichten zwar Wunderwerke an kreativer Spekulation und dichterischer Erzählkunst sind, aber was den Effekt betrifft, eher therapeutische Misserfolge. Heute, wo wir einige seiner therapeutisch gelungenen Fallgeschichten kennen gelernt haben, wie sie von Patienten überliefert sind, die kein propagandistisches Interesse an der Psychoanalyse hatten, ahnen wir, dass dies nicht ganz zufällig so ist. Freuds therapeutische Erfolge sind nämlich analytisch weit peinlicher als seine Misserfolge. Die von Pohlen publizierte Analyse von Ernst Blum beispielsweise oder die von Anna Koellreuter neulich am Psychoanalytischen Seminar Zürich (PSZ) vorgestellte Analyse ihrer Grossmutter zeigen Freud in seinen Deutungen als Analytiker der sich fast ausschliesslich und in uns eher banal anmutender Art für die ödipalen Verstrickungen seiner Patienten interessiert, aber sie weisen ihn als einen im persönlichen Umgang mit diesen sehr flexiblen und einfühlenden Menschen aus, (vgl. auch der von Martin Kuster aufgefundene Brief an Emmy von N's Tochter) der sich bestens auf die Kunst des Suggerierens verstand. 
Interventionen wie z. B. die Folgende sind pure Suggestion: «Sie streifen so nah am Geheimnis des untersten Stockes, dass ich es Ihnen verraten kann. Sie liebten Ihren Vater und haben ihm den Treuebruch mit der Mutter nie verziehen. Sie wollten die Mutter des Kindes sein. Sie wünschten daher Ihrer Mutter, die Ihnen den Geliebten nahm, den Tod. - Nach und nach werden Sie Beweise dazu bringen und es wird sich das Rätsel lösen, warum Sie nicht von Ihrem Bruder loskommen» (Kuster in diesem Heft). Diese Deutung machte Freud einer seiner Analysandinnen in einer der ersten Stunden! Diejenigen, denen solches gesagt wurde, hatten bereits sämtliche verfügbaren Schriften Freuds gelesen und es schmeichelte ihnen natürlich, dass der grosse Seelenforscher auch in ihren Biografien jene Strukturen erkannte, deren Beschreibung ihn berühmt gemacht hatte. Auf dem Hintergrund solcher Gratifikation konnte der zwänglerisch zweifelnde Ernst Blum seine Vorbehalte gegen seine nicht jüdische Verlobte beiseite legen und sie heiraten und konnte die von Skrupeln geplagte Frau G die Verlobung mit dem sie letztlich wenig interessierenden Arztkollegen auflösen und ihren Brienzer Bildhauer finden. Freud hatte, um es auf den kürzesten Nenner zu bringen, in diesen beiden Fällen - und sicher in vielen anderen auch - mittels seiner suggestiv eingesetzten Ödipusgeschichte die vor allem gesellschaftlich familiär bedingten Skrupel seiner Analysanden gegenüber einer freien Liebeswahl aufgelöst und dadurch neue Entscheidungsgrundlagen für diese Wahl ermöglicht, ohne die Wahl selbst suggestiv zu beeinflussen. (Dass «die Ödipusgeschichte» Freuds ureigenste Erfindung war, machte sie für ihn als Suggestionsvehikel so viel wirksamer als für seine Epigonen!) Das sind natürlich schöne Erfolge, die freundschaftliche Ratschläge zuvor nicht hatten bewirken können. Besonders tiefe Einsichten lagen ihnen aber nicht zu Grunde.

Warum also die asketische Haltung gegenüber der Suggestion, wenn doch diese so offensichtlich das therapeutische Agens der Veränderung ist? Freud war es als dem aufgeklärten modernen Geist, der er war, ein Gräuel, andere Menschen mittels undurchsichtigen Manipulationen zu verändern. Es geht hier nicht darum, eine, wie ich glaube falsche psychoanalytische Behauptung (Analyse sei frei von Suggestion) auf die Persönlichkeit Freuds oder gar seine Pathologie zurückzuführen. Die in dieser Scheu vor der Suggestion zum Vorschein kommende Haltung ist vielmehr ein Zug der ganzen Moderne und des aufklärerischen Weltbildes. Sie hat mit Freuds spezifischer Persönlichkeit zunächst wenig zu tun. Mit Freud ist das moderne Subjekt aufgebrochen, sein dunkelstes Innerstes zu erhellen. Es hoffte, dort auf Gesetzmässigkeiten zu stossen, die sein Funktionieren erklärten, es fand aber «nur» Motive irrationaler Art, die neben den bewussten unser Erleben und Handeln beeinflussen. Beeinflussen, hier haben wir den verflixten Terminus 
wieder, aber jetzt im Innern des Subjektes und nicht zwischen den Subjekten. Dieser Einfluss ist der Kontrolle des Ich entzogen, wirkt aber trotzdem auf dessen Erleben und Handeln. Könnte man die Gesetzmässigkeiten in Erfahrung bringen, nach denen da etwas Wirkung erzielt, so könnte man mittelbar Kontrolle über diese Einflussgrösse gewinnen, auch wenn sie unmittelbar nicht gemerkt, gespürt, gesehen wird, so wie wir über chemisch/physikalisches Wissen eine gewisse Kontrolle über Einflüsse auf unseren Körper gewinnen können, auch wenn wir diese Einflüsse nicht spüren. So können wir z. B. eine Vergiftung durch eine nicht gefährlich aussehende oder schlecht schmeckende Speise vermeiden oder eine unserer Gesundheit förderliche aber unangenehme körperliche Anstrengung trotz Widerwillen unternehmen.

Nun ist aber ein Motiv keine Ursache. Das heisst, um eine Wirkung zu erzielen, muss das Motiv erst von einer Instanz, z. B. dem Ich gewürdigt werden, um dann kraft eines Willensentscheides Grund für ein Erleben oder eine Handlung zu werden. Aber unbewusste Motive können nicht gewürdigt werden, da sie ja nicht zur beurteilenden Instanz des Ich vordringen. Sie müssen also entweder doch wie eine physikalische Ursache wirken, welche Möglichkeit wir ihnen soeben abgesprochen haben, oder sie müssen für eine andere Instanz als das Ich würdigbar sein und daraufhin Wirkung entfalten. Wer oder was aber wäre diese Instanz? Das ist die Gretchenfrage. Wenn ich aus Versehen die Vase zerschlage, welche ein Hochzeitsgeschenk der verhassten Tante Emma war, wer hat dann gestossen? Das Es werden Sie als Psychoanalytiker sagen, aber mit dieser so schönen Antwort kommen wir nun zumindest in zwei beträchtliche Schwierigkeiten, die meist zu wenig bedacht werden. Erstens fehlt dem Es der psychoanalytischen Metapsychologie, das keine Struktur hat, alles, was es befähigen könnte, etwas zu würdigen, ein Urteil zu fällen und zweitens fehlt dem Es jene schillernde Zweideutigkeit, die das Ich auszeichnet, indem dieses nämlich einerseits - im alltagssprachlichen Gebrauch - ein unmittelbar erfahrbarer Erlebensinhalt ist, andererseits aber ein theoretisches Konstrukt, das mit dem ersteren nicht identisch ist. Wenn wir einen Satz sagen, wie «das Ich fügt sich dem Druck der Realität», dann hilft uns diese Zweideutigkeit, denn ich bin es ja, der sich in der Erfahrung diesem Druck beugt und nicht ein Ich in mir, wenn ich aber sage, mein Es habe die Vase zerschlagen, so ist das eine schwer akzeptable abstrakte Behauptung, denn auch hier bin es doch ich offensichtlich gewesen, der gehandelt hat. Es fehlt uns also in der psychoanalytischen Terminologie ein Begriff, der jene Instanz bezeichnen würde, die zwar handeln kann im Sinne von Urteile fällen, Entschlüsse fassen usw., die aber nicht mit dem Ich zusammenfällt und deshalb sowohl in seinem Sinne, wie auch 
diesem entgegengesetzt handeln kann. Ein solcher Begriff ist z. B. die Person oder das Subjekt, aber beide sind der ursprünglichen psychoanalytischen Theorie fremd und haben dementsprechend in ihrer Metapsychologie keinen Ort.

Ein Subjekt untersteht verschiedenen, sich nicht deckenden Einflussgrössen. Einerseits sind da die seinem Ich bewusst zugänglichen, eigener Einsicht entoder auch widersprechenden von aussen kommenden Erwartungen, Gesetze und Anforderungen und andererseits sind es die in seinem Es aufbewahrten, von anderen stammenden, nicht ichgerecht übersetzten Botschaften, die uns in unserem Handeln und Erleben treiben, aber etwas ganz anderes sind, als die biologischen Instinkte, die Triebe der Biologie. Wo diese Anforderungen verschiedenen Ursprungs sich nicht decken, sind wir zwischen ihnen hin und her gerissen. Da mag das Ich sich entscheiden wie es will, das garantiert noch lange nicht, dass sich das Subjekt diesem Entscheid anschliesst. Leider aber gibt es keine Gesetzmässigkeit, nach der sich dieser Konflikt regelhaft entscheidet. Der Ausgang ist vielmehr kontingent und muss es auch sein, damit bei den je nachdem selteneren oder häufigeren Siegen des Ich so etwas wie ein Gefühl von Freiheit entstehen kann. Aber diese Freiheit ist immer eine nur vorübergehend geschenkte und niemals eine garantierte. Es kann jederzeit genau so gut der nicht bewusst wahrnehmbare andere in mir (nicht in meinem Ich!) die Oberhand gewinnen.

Wenn es uns in der Analyse gelingt, ein unbewusstes Motiv aufzuklären, indem wir es historisch herleiten, so ist dieses damit nicht ausser Kraft gesetzt. Wenn sich z. B. die perverse Neigung, sich masochistisch zu unterwerfen, sich im Leben Prügel einzuhandeln oder die sexuelle Lust an das Geschlagenwerden zu koppeln als nicht erkannter Gehorsam gegenüber dem unausgesprochenen väterlichen Gebot «Nur wer geschlagen wird, wird geliebt» herausstellt, so verliert deshalb dieses Geschlagenwerden keineswegs von selbst seine Attraktion. Es kann diese verlieren, aber nur unter der Bedingung, dass die Liebe und Anhänglichkeit an jenen, der diese Entdeckung ermöglicht hat, an den Analytiker/Therapeuten, wirkmächtiger wird als jene an den Vater. Und das ist weder mit der Korrektheit des Inhalts der Deutung garantiert, noch durch deren technisch richtige Applikation, sondern ist allein ein Erfolg der im Medium dieser Deutung mitlaufenden Suggestion. Dieser Suggestionserfolg aber kann, so sehr uns dies kränken mag, auch auf ganz andere Art herbeigeführt werden, z. B. durch ein inbrünstiges gemeinsames Gebet, durch ein Gruppenritual, eine zeremoniell verspeiste Substanz, eine in Aussicht gestellte Prämie, einen überraschend hergestellten Zusammenhang, auch wenn er bloss fiktiv ist und was der Tricks der Seelsorger, Therapeuten, Medizinmänner, Pädagogen, Schamanen und Esoteriker mehr sein mögen. Dieser Suggestionserfolg kann aber 
auch ausbleiben, wie «richtig» die Deutung immer war, wie inbrünstig das Gebet und wie eindrücklich das Ritual.

Der langen Rede kurzer Sinn: Eine bei anderen herbeigeführte Veränderung ist immer das Resultat von Suggestion. Einsicht - wahre oder falsche - kann ein Medium von Suggestion sein, muss es aber nicht. Und Suggestion bedeutet immer einen Einbruch in das, was wir Autonomie nennen, denn sie wirkt auf eine Weise, die sich der Kontrolle des Subjektes entzieht, nämlich über Liebe oder psychoanalytisch ausgedrückt, über die Sexualität im erweiterten Sinne, über das Triebhafte, jenen Teil, der uns in nicht abgrenzbarer Weise mit anderen verbindet. Die «Liebe» (und natürlich auch ihr Gegenteil, der Hass) ist also sowohl Garant unserer Freiheit, wie auch deren Begrenzung. Unter ihrem Einfluss können wir uns anders verhalten, als nach den «Gesetzmässigkeiten» unseres Ich zu erwarten wäre, aber diese Freiheit bedeutet, so paradox dies erscheinen mag, gleichzeitig Aufhebung der Autonomie.

In der politisch korrekten Terminologie unserer Tage müsste man eigentlich sagen: Suggestion ist grundsätzlich immer Missbrauch, denn sie unterläuft die autonome Entscheidungsfreiheit des anderen, wenn auch, wie die Therapeuten bona fide annehmen, in dessen Sinne und zu seinen Gunsten. Aber das nehmen eben viele, die wir heute als gewöhnliche sexuelle Verführer einstufen, auch an. Es gibt allerdings eine elegante Möglichkeit, diesen «Missbrauch» zu beschönigen, nämlich die Einhaltung der Abstinenz, verstanden im engeren Sinne als Abstinenz von absichtlicher Suggestion. Solche Abstinenz darf man als gegeben vermuten, wenn der Analytiker bewusst von allem absieht (unbewusst kann er das natürlich nicht), was Suggestion sein könnte und sich bei seinen Interventionen nur vom Gesichtspunkt der Einsichtsgewinnung leiten lässt. Das war Freuds ursprüngliche Idee, als er sich von der Hypnose abwandte, allerdings verbunden mit der irrtümlichen Annahme, das führe dann von selbst zur Veränderung. Sein späterer Pessimismus bezog sich auf die Einsicht, dass es das nicht tut. Abstinenz in diesem Sinne ist also eine Art Alibi des Therapeuten, der Trick mittels dessen er sich der Einsicht verschliesst, dass seine Deutung suggestiv war und dieses Alibi ist kaum überzeugender als jeder andere entschuldigende Hinweis des Inhaltes «das war mir nicht bewusst.» Im Allgemeinen ist ja gerade Analytikern eine solche Ausrede höchst verdächtig.

Man muss allerdings weder ein Pessimist noch ein Betrüger sein, um die Analyse in diesem ursprünglichen Freudschen Sinne zu betreiben und zu vertreten, so wie ich das aus Überzeugung tue, denn das Erlangen von Einsicht hat unabhängig davon, ob es sonst noch etwas bewirkt und was es bewirkt, einen Wert an 
sich, bedeutet es doch, fern von aller Zweckrationalität Lust, steht also im Dienste der Sexualität jenseits deren biologischer Funktionalität. Erkenntnis, die ja auch in der altertümlichen deutschen Sprache identisch ist mit sexuellem Verkehr, ist Lust an sich und bedarf keiner funktionalen Begründung (dass sie gesund macht, reich oder erfolgreich oder begehrenswert). Echte Forschung steht nicht, wie es uns unsere Politiker, die Wirtschaftsbosse und selbst viele Professoren glauben machen wollen, in erster Linie im Dienste des gesellschaftlichen und technologischen Fortschritts. Sie ist vielmehr die Nachfahrin der kindlichen Sexualneugier und ihr Antrieb ist genau wie bei jener das vage Versprechen von Lust, die sich im Erfolgsfalle mindestens als Stolz und Genugtuung einstellt. Und etwas damit nahe verwandtes ist auch das erklärte Ziel der Analyse, wie ich sie verstehe im Unterschied zur Therapie, deren erklärtes ZielVeränderung ist. Nur in diesem Sinne macht für mich das viel bemühte Junktim von Forschen und Heilen einen Sinn. Es kann nicht heissen, man müsse implizit wissenschaftliche Therapieforschung betreiben, sondern, man könne nichts Unbewusstes zum Vorschein bringen, wenn man nicht mit jener ursprünglichen Forscherlust, die ein direkter Abkömmling der kindlichen Sexualneugier ist, zu Werke geht, was voraussetzt, dass man stets aufs Neue alles kodifizierte Wissen suspendiert. Und deshalb eben war Freud mit seiner Ödipusgeschichte, die er bei jedem Analysanden wieder neu er-fand, suggestiv erfolgreicher, als viele, die sie aus dem Lehrbuch nachbeten, ohne sie je selbst neu erfunden zu haben

Dass Einsicht und Veränderung zusammenfallen können, ist ein willkommener Bonus. Und Sie haben natürlich auch recht, wenn Sie mir entgegenhalten, jede Einsicht bedeute an sich immer schon eine Veränderung, denn derWissende sei nicht mehr der gleiche, wie der vorher Unwissende und überdies hätten wohl sehr viele Veränderungen sehr viel mit Einsicht zu tun. Natürlich kommt niemand nur um der Erkenntnis willen in eine Analyse, aber derjenige, der wirklich Analyse begehrt, schätzt Veränderung, die sich auf Grund von Einsicht ergibt höher, als jede andere Veränderung, genau so wie jener, der die Welt bereist, um mehr zu wissen, dieses so erworbene Wissen, das aus eigener Erfahrung entsprungen ist, in anderer Weise besetzt, als dasjenige, welches er aus Büchern bezieht. Man kann Einsicht und Veränderung nicht total auseinanderdividieren, aber ich glaube, man macht es sich auch zu leicht, wenn man sie einfach in jenem dialektischen Verhältnis sieht, das wir so gerne aus dem Zylinder zaubern, wenn wir in Widersprüche zu geraten drohen. Es gibt nicht nur dialektische Verhältnisse, sondern auch Unterschiede, Differenzen, und diese lassen sich nicht dialektisch auflösen. 
Warum, so werden Sie mit Recht fragen, soll man nun so viel Wert auf diese Unterschiede legen, wenn man doch nicht bestreitet, dass jenseits von ihnen vieles, ja sogar das meiste in einem analytischen und einem therapeutischen Prozess gleich ist. Dafür gibt es, wie ich meine, mindestens zwei gute Gründe. Den ersten könnte man einen ästhetischen und subjektiven nennen. Eine der zentralsten und weitest reichenden Entdeckungen der Psychoanalyse besteht darin, dass sie an der menschlichen Sexualität einen Aspekt freigelegt hat, der diese wesentlich charakterisiert und von der animalischen Sexualität unterscheidet, nämlich ihre Disfunktionalität. Damit ist die Tatsache gemeint, dass sie im Unterschied zur der nur der Arterhaltung dienenden, instinktiven Geschlechtlichkeit, funktionslos ist, bzw. in erster Linie der Lust dient und in dieser von aller natürlichen Funktionalität abgekoppelten Selbstgenügsamkeit den Kern der menschlichen Kultur und der spezifisch menschlichen Psyche ausmacht. Solche Einsicht konnte sich nur in einer Forschungsanlage zur Darstellung bringen, die ihrerseits von allem Zweckhaften und Funktionalen absah, eben im analytischen, allein von der freien Assoziation und der freischwebenden Aufmerksamkeit geleiteten Dialog. Es wäre deshalb ein unersetzlicher Verlust, würde man diesen spezifischen Dialog nicht weiter pflegen und ihn zu einer historischen Reminiszenz eindampfen, nur weil man nicht wissen kann, ob er auch in Zukunft noch Quelle neuer und bedeutungsvoller Einsichten sein wird. Sie mögen das als eine denkmalschützerische Betrachtungsweise qualifizieren, aber dann sage ich Ihnen, dass ich ganz entschieden für den Denkmalsschutz bin.

Der zweite Grund ist pragmatischer und hat durchaus mit den recht verstandenen Interessen der Psychotherapie, wenn auch nicht notwendig mit den standespolitischen der Psychotherapeuten zu tun. Da die Ausrichtung der Therapie und der Analyse verschieden sind, braucht es auch verschiedene Wege zur Aneignung der beiden Kompetenzen. Diese mögen sich in weiten Bereichen decken, aber in gewissen hoch spezifischen tun sie das nicht, ja sie können sich dort u. U. gegenseitig schwer beeinträchtigen. Die strategischen Überlegungen z. B., die notwendig sind, um sicherzustellen, dass der therapeutische Prozess auf kürzestem und wirksamstem Weg zu den vom Patienten gewünschten Veränderungen führt, sind Gift für den analytischen Prozess. Solch strategische Ausrichtung bedingt eine ständige Selektion in der Wahrnehmung des Therapeuten, die dazu führt, dass all das Nebensächliche, Redundante, Unscheinbare, Nutzlose, Disfunktionale untergeht, welches gerade den Lauf des verschlungenen analytischen Pfades bestimmt. Kurz, das Unbewusste kann nur in den Blick kommen, wo keine 
Strategie auf etwas aus ist, was der Therapeut, der seinem Wesen nach ein Experte ist, schon weiss. Andererseits ist die freischwebende Aufmerksamkeit des Analytikers, die im Extremfall dazu führt, dass man, wie Conrad Stein das vorgeschlagen hat, in jede Sitzung so hinein geht, als wüsste man nichts, ein Hohn angesichts des Wunsches des Patienten nach schnellstmöglicherVeränderung und der Zumutung, dass ein öffentlicher Kostenträger, der auf dem Solidaritätsprinzip gründet, für dieses Unterfangen bezahlen soll. Die Abstinenz vom (immer schon) Wissen macht also den grossen Unterschied aus. Der psychoanalytische Therapeut benutzt wie jeder andere Therapeut sein psychoanalytisches Wissen über die Psyche, um gezielt an etwas zu arbeiten - und das ist seine oberste Leitlinie-, während der Analytiker dieses Wissen systematisch immer wieder «vergisst», suspendiert, um das zu sehen und zu hören, was man sonst nur durch die Risse dieses Wissens hindurch andeutungsweise sehen und hören kann, das Unbewusste. Diese Haltung ist nicht nur heute unter Analytikern recht selten, sondern sie war auch bei Freud mehr ein Postulat, als dass er sie in seinen Kuren, die oft mehr Therapien oder Lehrgängen ähnelten, wirklich befolgt hätte. Das muss aber nicht hindern, sie sich als nie voll verwirklichbares Ideal beim Analysieren vor Augen zu halten.

Man kann selbstverständlich mit allen analytischen Konzepten auch therapieren. Jene ganz und gar bewusstseinsnahe Übertragung etwa, von der heute auch der properste Verhaltenstherapeut andauernd redet und die in der schlichten Tatsache besteht, dass der Patient erstens zu seinem Therapeuten eine spezifische Beziehung hat und diese zweitens in irgend einer Weise an die Beziehungen zu den grossen Figuren seiner Vergangenheit anlehnt, hat wenig mit jener spezifischen unbewussten Übertragung der Psychoanalyse zu tun, die in einer unbemerkten falschen Verknüpfung besteht, die es manchmal aufzulösen, manchmal auch schlicht zu beachten gilt. Denken Sie an das legendäre Schwerhörigen-Beispiel von Morgenthaler (Morgenthaler 198162 f), wo es nicht darum ging, dem Analysanden zu sagen, er verwechsle den Analytiker mit dem Vater, weil er diesen anstatt jenen für schwerhörig halte, sondern lediglich darum, darauf hinzuweisen, dass der Analytiker nicht schwerhörig sei, also eine Bindung aufzulösen und nicht eine neue herzustellen, in der sich sogleich alles wieder neu verfestigt. Morgenthaler sprach deshalb mit Vorliebe davon, dass es darum gehe, das Befremdliche, Störende in einer ansonsten guten Beziehung hervorzuheben und nicht darum, es mit vorschnellen Erklärungen zum Verschwinden zu bringen. Übertragung ist etwas Ubiquitäres und in ihrer bewusstseinsnahen Ausformung ein wichtiges Ingredienz jeder Psychotherapie, aber auch jedes Meister/Schülerverhältnisses und jeder Liebesbeziehung, als unbewusste aber etwas exquisit Psychoanalytisches bzw. nur 
in der Psychoanalyse Beachtetes und Gewürdigtes. Die unbewusste Übertragung etwa meines Analysanden mit der bizarren Onaniephantasie zeigte sich in den wiederholten Fehlleistungen des Verpassens der Busstation bei meiner Praxis, nachdem ich ihm diese Phantasie «entlockt» hatte und auf ihren Zusammenhang mit der befürchteten Strafe der Mutter für seine Sünde hinwies. In der Fehlleistung manifestierte sich seine ubw. Tendenz, vor mir Reissaus zu nehmen, weil ich ihm eben unbewusst - gerade dort, wo ich mich ihm interessiert zugewandt hatte, nun erschien wie seine Mutter, deren Zuwendung zugleich immer auch Einengung und Behinderung seiner triebhaften Wünsche bedeutet hatte. Die bewusste und wie ich meine unspezifische Übertragung dagegen war auch in dieser Behandlung jene wohlwollend positive Grundhaltung mir gegenüber, die das ganze in Gang hielt und in der mich der Analysand in Anlehnung an den verehrten, aber meist abwesenden Vater wahrnahm, der ihn aus dem Gefängnis der Mutter hätte befreien können, aber es nicht getan hatte.

Nun könnten Sie mich nicht ohne Grund fragen, wieso ich eigentlich so heftig für die Analyse und gegen deren Aufgehen in der Therapie und nicht vielmehr für die Therapie und deren Ausbau und Fortschritt votiere, denn die Therapie sei ja doch ohne Zweifel die nützlichere Sache und diejenige, auf die eine Gesellschaft, der an der psychischen Gesundheit ihrer Mitglieder liege, auf keinen Fall verzichten könne. Die Analyse, wie ich sie darstelle, erscheine dagegen eher als eine ästhetische Angelegenheit, ein Produkt entstanden aus einer besonderen gesellschaftlichen Konstellation, die ihren Beitrag zur menschlichen Kultur geleistet hat und auf die man, mindestens als jene Praxis auf der Couch, in der es in erster Linie um das Analysieren und Entbinden geht, auch verzichten könnte. Dem würde ich grundsätzlich nicht widersprechen, sondern bestätigend hinzufügen, dass es so etwas wie Psychotherapie schon immer gegeben hat, auch wenn das unter anderen Titeln wie Seelsorge, Magie, religiöse Exerzitien u.s.w. subsumiert worden sein mag, und dass es so etwas auch in Zukunft geben wird. Wenn ich selbst Therapien mache, dann mache ich das nicht aus blosser Verlegenheit, weil es zu einer Analyse nicht reicht, sondern aus innerer Überzeugung und aus Respekt vor den Patienten, die dies und nichts anderes wünschen.

Die Analyse ist in ihrer klassischen Form im Wesentlichen eine Erfindung, die zum Anfang des zwanzigsten Jahrhunderts gehört. Man muss auch sich selbst und das, was man tut, in einer historischen Dimension sehen und nicht immer nur das, was die anderen tun und was man ablehnt und als überholt beseitigen möchte, so wie das gewisse Vulgärmarxisten mit Vorliebe tun. Was ich befürchte ist nicht, dass wir alle zu Therapeuten werden - das sind wir ohnehin - sondern, 
dass die eigentliche Analyse ganz langsam in der zeitgemässen Therapie aufgeht und verschwindet. Was man aus ihr gebrauchen kann, die Kenntnisse über die Dynamik des Unbewussten, die Metaphorik des psychischen Apparates und der Instanzenlehre, eine eh problematische Entwicklungspsychologie mit ihren putzigen Stadien, das wird man beibehalten und in der Therapie gezielt einsetzen. Auch das Insgesamt der Theorie wird als ein Kapitel der Philosophie und als ein nützliches tool in der Kunst- und Literaturwissenschaft seinen Platz behalten. Ich stehe auch nicht an, freimütig zu bekennen, dass für mich persönlich das Wichtigste an der Psychoanalyse ihre anthropologische Dimension ist, das heisst, ihre explikative Kraft hinsichtlich des Verständnisses dessen, was der Mensch ist. Trotzdem aber hänge ich ebenso sehr an der von dieser Theorie inspirierten Praxis auf und hinter der Couch. Und diese nicht primär therapeutische Analyse, die durch nichts anderes definiert ist, als durch ihre innere Ausrichtung, die dialogische Selbsterfahrung, welche um der Erkenntnis willen, alle Konsistenz in der Rede des Analysanden destruiert, droht auf dem grossen Marktplatz der immer neuen spektakulären, erlebnis- und nicht erkenntniszentrierten Selbsterfahrungsmoden und in der Konkurrenz mit den stets hochaktuellen störungsspezifischen modernen Therapieformen zu einer unbedeutenden Marginalie zu verkommen und das finde ich schade. Neben dem Psychotherapiecurriculum gibt es sie am PSZ dann zwar immer noch, aber man weiss nicht recht wo. Dieses Curriculum und die berufspolitischen Probleme der Psychotherapeuten beherrschen schon heute sowohl die Internetsite, wie auch die Teilnehmerversammlung und die Arbeit der Seminarleitung immer mehr. Weiterbildung ist angesagt. Die psychoanalytische Ausbildung, die schon immer paradoxe Züge hatte, weil sie eher eine Bildung ist, denn eine Aus- oder gar Weiterbildung, ist zugleich überall ein bisschen und nirgends recht und wird immer mehr zur historischen Reminiszenz, ein bisschen so wie der geflügelte Genius auf dem Kühler eines Luxusprotzes von BMW nur noch eine Reminiszenz ist an jene Edelkarossen, die einst auf den Strassen Zeugnis von der Ingenieurskunst britischer Flugzeugmotorenhersteller ablegten.

Was wir benötigen, das schützen wir, wenn es uns bedroht erscheint, selbst wenn wir vorübergehend vielleicht nicht bemerken, dass wir es schädigen. Aber irgend einmal wird das offensichtlich und dann wird, wie wir wissen auch die SVP grün und dann fördert auch die Atomlobby die Entwicklung alternativer Technologien, aber so lange es noch anders geht, sollen das doch die Idealisten auf eigene Rechnung tun. «Wir steigen dann ein, wenn es rentabel wird.» Um die Zukunft der Psychotherapie ist mir deshalb nicht bange. Sie wird auch von Leuten, wie Herrn Couchepin schlussendlich garantiert werden, denn es braucht sie. Aber 
ich bin eben nicht für den Schutz des Waldes, weil er die grüne Lunge unseres Planeten ist, sondern weil er uns zu romantischen Liedern und Bildern inspiriert und weil man dort Herzen in die Rinde von Bäumen schnitzen kann - durchaus kein ökologisch sinnvoller Vorgang. Und ich bin als Denkmalschützer - ich habe es schon gesagt -, nicht für die Erhaltung der alten Kerne unserer Städte, weil sie touristisch von unschätzbarem Wert sind, sondern weil sie uns daran erinnern, wie es ehedem war und weil es zu meinem Konzept von Humanität gehört, dass wir solche Erinnerungen um ihrer selbst willen hoch halten. Die Psychoanalyse auf der Couch vermittelt eine Erfahrung, die aus den Bedürfnissen und Wünschen des modernen Menschen des ausgehenden 19. und des beginnenden zwanzigsten Jahrhunderts entsprungen ist, der sich einen Ort jenseits aller funktionalen Zwänge und aller ichbeherrschten Disziplinen erträumte, der sich für das Andere in ihm und jene Anderen vor ihm interessierte, die aus ihm sprachen, just wenn er selbst zu sprechen glaubte. Er wollte Stimmen aus seinem Inneren zu Gehör bringen, die vom anschwellenden Lärm der Aussenwelt jener Epoche immer mehr übertönt wurden und die man nur vernehmen kann, wenn man all das ideologische und geschäftige Geschwätz zum Verstummen bringt. Er wollte nicht normal sein, im Sinne eines "Comme il faut», sondern wissen, warum er gerade so ist, wie er ist. Es ist wohl möglich, dass dies dem postmodernen Menschen des einundzwanzigsten Jahrhunderts weniger ein Bedürfnis ist, sei es, weil er sich an diesen Lärm gewöhnt hat und ihn zuweilen gar für Musik hält, sei es, weil die Funktionalitäten längst solche Herrschaft über ihn gewonnen haben, dass er sie als solche gar nicht mehr störend wahrnimmt, - wir wollen doch gut funktionieren - sei es schliesslich, dass er vor allem dank des Inputs der Medien in so ununterbrochenem Austausch mit Anderen und Anderem steht, dass die Wiederbelebung der Stimmen der Anderen aus der Periode seiner persönlichen Menschwerdung, keinen so besonderen Reiz mehr hat. Vieles verliert durch veränderte historische Bedingungen an Bedeutung, so z. B. in unserem Telekommunikationszeitalter das Briefeschreiben. Ich glaube aber, dass gewisse Erfahrungsformen, die geschichtlich bedingte Blüten kannten, wie z. B. die Malerei in der Renaissance und im 19. Jh. oder das Briefeschreiben im Zeitalter der Romantik auch dann weiter gepflegt werden sollten, wenn sie nicht mehr als zentrale Anliegen erscheinen. Auch wenn die bildenden Künste heute in derVielfalt neuer Medien unbegrenzte, unverbrauchte Artikulationsmöglichkeiten finden, sollte man trotzdem weiter Tafelbilder malen und diese Kunst auch lehren, und trotz SMS sollte man sich nicht völlig vom geschriebenen Brief entwöhnen, weil in beiden Ausdrucks- und Mitteilungsformen eine wie auch immer historisch bedingte Form menschlicher Erfahrung liegt, ohne deren Kenntnis und Pflege wir 
ärmer wären, wenn auch nicht im ökonomischen Sinne. Schützen und hegen muss man eben die Dinge, deren Existenz durch keine Funktionalität, keinen materiellen Gewinn und keine Mehrheitsbedürfnisse garantiert ist, z. B. die Schnabeltiere, die Lyrik und die Psychoanalyse.

\section{Literatur}

Freud, S. (1917): Eine Schwierigkeit der Psychoanalyse, GW Bd. XII (1952). Frankfurt a. M.: Fischer Verlag, .

Freud, S. (1925): Einige Nachträge zum Ganzen der Traumdeutung, GW Bd. I (1952). Frankfurt a. M.: Fischer Verlag, .

Koellreuter, A. (2007): Als Patientin bei Freud 1921 - Aus dem Tagebuch einer Analysandin. In: Werkblatt 58, 3-23.

Kuster, M. (2008): «Ich persönlich werde wohl die «klassischen`Analysen weitermachen». In diesem Heft.

Laplanche, J. (1996): Die unvollendete Kopernikanische Revolution in der Psychoanalyse. Frankfurt a. M.: Fischer Verlag.

Laplanche, J. (2007): Sexual. La sexualité élargie au sens Freudien. Paris: puf.

Morgenthaler, F. (1981): Technik. Zur Dialektik der psychoanalytischen Praxis. Frankfurt a. M.: Syndikat Verlag. 